\title{
Modeling of multicomponent diffraction structures based on optimization approaches and numerical methods
}

\author{
Igor Lvovich ${ }^{1}$, Yakov Lvovich ${ }^{2}$, Andrey Preobrazhenskiy ${ }^{1, *}$ and Oleg Choporov ${ }^{2}$ \\ ${ }^{1}$ Information Systems And Technologies Department, Voronezh institute of high technologies, 73a, \\ Lenina st, Voronezh, 394043, Russia \\ ${ }^{2}$ Information Security Department, Voronezh state technical university, 14, Moscow dist., Voronezh, \\ 394026, Russia
}

\begin{abstract}
The paper proposes a methodological approach in which the representation of objects in the form of a set of diffraction structures is their Association into groups. Using neural network modeling, expert evaluation and application of optimization based on genetic algorithm, there is a formation of the object with the desired scattering properties. An example of modeling an object presented as a set of two-dimensional cylinders is given.
\end{abstract}

\section{Introduction}

The problems of creating electrodynamic objects with specified scattering properties have long been considered by various researchers $[1,2]$. Objects have, as a rule, a complex form [3] and it is impossible to say that there are universal methods of calculation and optimization of scattering properties.

In this paper, it is proposed to provide the required scattering characteristics based on the representation of objects as groups of diffraction structures and the use of a combination of optimization approaches [4].

\section{Objects of research}

Objects having a complex shape are represented as a set of diffraction structures (DS) $D_{i}$, $(i=1, . ., \mathrm{I})$ each of them characterized by a vector of indices of the scattering properties (the radar cross section (RCS)) $\sigma_{i}$. In this case

$$
\sigma_{i}=\left(\sigma_{i 1}, \ldots, \sigma_{i j}, \ldots, \sigma_{i J}\right) \text {. }
$$

DS are combined into groups $(m=1, . ., \mathrm{M})$ to provide the required scattering levels for the entire object. Objects are grouped into groups so that the scattering level in the

\footnotetext{
* Corresponding author: komkovvivt@yandex.ru
} 
$(m-1)$-th group is greater than in the $m$-th. We consider optimization models and numerical methods for creating optimal objects with respect to two components: structural and scattered field levels. The structure is improved by the transformation of $D_{i}(i=1, . ., \mathrm{I})$ to another set $D_{i_{1}},\left(i_{1}=1, . ., \mathrm{I}_{1}\right)$ here $I_{1}<I$, and the level of the scattered field due to the displacement DS of the groups are more integrated RCS in the group with less its value. Different approaches are used to estimate the integral RCS of estimation. For example, this model can be used:

$$
Y_{i}=F\left(\sigma_{i j}\right)=\min _{1 \leq j \leq J} \alpha_{j} \sigma_{i j} \text {, where } \alpha_{j}>0, \sum_{j=1}^{J} \alpha_{j}=1 \text {. }
$$

It will correspond to the value of the lowest value of the scattered electromagnetic field on the DS $D_{i}$.

\section{Neural network modeling of DS group creation}

In order to solve the problem of grouping DS characterized by RCS $\sigma_{i}$, we will consider two specific neural networks that belong to the ART family. In obtaining a set of groups that consist of DS having similar sets of performance indicators, we proposed a combination of such networks: ART-2A Network [5] and Furry ART Network [6]. If the prototype of the group satisfies the similarity condition, we train such a prototype by changing its weights so that it is more similar to the input vector. Normalize the initial vectors $\sigma_{i}(i=\overline{1, I})$. We apply the cosine function of the angle between the corresponding vectors, it is characterized by their scalar product

$$
T_{k}=M_{k}=\left(\sigma_{i}, w^{k}\right)=\sum_{j=1}^{J} \sigma_{i j} w_{i}^{k}
$$

here $T_{k}$ shows the value of the selection function for the $k$-th group, and $M_{k}$ - the value of the matching function, $w^{k}-$ is the current prototype vector for the $k$-th group. If the prototype of the group satisfies the similarity condition, we train such a prototype by changing its weights so that it is more similar to the input vector:

$$
w^{k+1}=\frac{(1-\beta) w^{k}+\beta \cdot \sigma_{i}}{\left\|(1-\beta) w^{k}+\beta \cdot \sigma_{i}\right\|},
$$

here $\beta$ is a parameter showing the speed during training.

For the grouping process to be switched to the Fuzzy ART network, it is necessary for the data to scale within $[0,1]$. The network is controlled by the parameters $\alpha, \beta, \rho$, here $\beta$ and $\rho$ are used by analogy with the ART-2a network, and $\alpha$ is an ultra-small number (its order is $\left.10-^{6}\right)$, which prevents the prototypes from completely degenerating. Selection function:

$$
T_{k}=\frac{\left|y_{i} \wedge w^{k}\right|}{\alpha+\left|w^{k}\right|}
$$


where the operator ${ }^{\wedge}$ is defined as $(p \wedge q)_{i}=\min \left(p_{i}, q_{i}\right)$, and the norm $|p|=\sum_{i} p_{i}$

Match Function:

$$
M_{s}=\frac{\left|\sigma_{i} \wedge w^{s}\right|}{\left|\sigma_{i}\right|}
$$

We select the group when the condition $M_{s}>=\rho$, is fulfilled, where $\rho$ - is an integral criterion for similarity. If the condition is violated, the group is marked as inactive and the selection function is called again. For the training function: the modification of the weights of the tested prototypes goes like this

$$
w^{t+1}=(1-\beta) w^{t}+\beta\left(\sigma_{i} \wedge w^{t}\right) .
$$

That is, when divided into groups, the space of input vectors will be covered with ndimensional parallelepipeds, as a prototype in each cluster there will be a parallelepiped that is closest to its center in the sense of Euclidean.

\section{Expert-optimization modeling of partitioning into groups of DS}

We suppose that based on the results of the evaluation, the DS was ranked relative to the integral RCS Y, which is normalized to the numerical interval [A, O]. Then the experts set the number of groups $(M)$ into which it is necessary to divide the whole set of DS and boundary values into two adjacent $(m-1)$-th and $m$-th groups, which are proportional to the maximum possible value of the integral RCS A:

$$
\mathrm{Y}_{\mathrm{m}-1, \mathrm{~m}}=\chi_{\mathrm{m}-1, \mathrm{~m}} \mathrm{~A}
$$

where $\chi_{\mathrm{m}-1, \mathrm{~m}}$ - is the proportionality coefficient, which is introduced by experts to separate the $(m-1)$-th and $m$-th groups, $0<\chi_{\mathrm{m}-1, \mathrm{~m}}<1$. In order to verify the normative separation of many diffraction structures into $m$ groups, we propose to form an optimization model.

Suppose that when we divide by condition (7), the DS $D_{i m-1, m}\left(i_{m-1, m}=\overline{1, I_{m-1, m}}\right)$ consists of the $(m-1)$-th and $m$-th groups. Optimization modeling is based on the fact that alternative (Boolean) variables are introduced [7]:

$$
x_{i}=\left\{\begin{array}{c}
1, \text { if the } i-t h \text { DS should be assigned to }(m-1)-\text { th group, } \\
0, \text { othewise, } \\
i_{m-1, m}=\overline{1, I_{m-1, m} .}
\end{array}\right.
$$

In this case, the choice according to the values of variables (8) should be made so that in the group with a higher level there are DS with lower values of the integral RCS. On the other hand, from the set of RCS values $\sigma_{j} \quad(j=\overline{1, J})$, basic values $s=\overline{1, S}$ are determined, with respect to which threshold values $\sigma_{s}^{n} \quad(s=\overline{1, S})$ are set, and for each $i-$ th DS, we form a set of binary estimates 


$$
c_{s i}=\left\{\begin{array}{l}
1, \text { if } \sigma_{i s} \geq \sigma_{s}^{n} \\
0, \hat{a} \text { otherwise } \\
s=1, S
\end{array}\right.
$$

When separating groups of DS, the condition is used

$$
\sum_{s=1}^{S} c_{s i}>c_{m-1, m}<\frac{1}{2} S .
$$

In the optimization model, the choice of a DS with lower values of the integral EPR is based on optimization criteria, and condition (9) is taken into account in the form of restrictions:

$$
\begin{gathered}
\sum_{i=1}^{i, m} \sigma_{i} x_{i} \rightarrow \min \\
\sum_{s=1}^{S} c_{s i} x_{i} \geq c_{m-1, m}, i=\overline{1, I_{m-1, m}}, \\
x_{i}= \begin{cases}1, & i=\overline{1, I_{m-1, m}} . \\
0, & \end{cases}
\end{gathered}
$$

It is possible to solve problem (10) using a directed randomized search using probability estimates $\tilde{x}_{i}$ of variables (8):

$$
P\left(\tilde{x}_{i}=1\right)=p_{x_{i}}, P\left(\tilde{x}_{i}=0\right)=q_{x_{i}}, p_{x_{i}}+q_{x_{i}}=1
$$

here $\mathrm{P}(\cdot)$ - indicates the probability of the event, $p_{x_{i}}$ - the probability values of the event $\tilde{x}_{i}=1, q_{x_{i}}$ - the probability value of the event $\tilde{x}_{i}=0$. When searching at each iteration, we achieve that the condition for local improvement is fulfilled. As a result, the probability values $p_{x_{i}}$ of part of the variables change from 0.5 to the values that fall into the neighborhood $(1-\varepsilon, 1)$, where $0.5>\varepsilon>0$, and the other part - into the neighborhood $(0, \varepsilon)$.

In the optimization model, the choice of DS with lower values of the integral RCS is based on optimization criteria, and the problem is solved by using directional randomized search using probabilistic estimates of variables.

The separation of objects between the $(m-1)$-th $m$-th groups does not coincide with the normative separation of experts from the managing center, and a compromise solution is required. To this end, it is proposed to use the mechanism of visual-figurative intuition of an expert [8].

In this case, a visual-figurative model of the set of objects $\sigma_{m-1, m}\left(i_{m-1, m}=\overline{1, I_{m-1, m}}\right)$ is formed in the form of an image (visualization) of multidimensional monitoring estimates, $\sigma_{i, j}, i=\overline{1, I_{m-1, m}}, j=\overline{1, J}\left(i_{m-1, m}=\overline{1, I_{m-1, m}}\right)$ by individual points on the plane [8]. 
Based on the location of the points, the expert draws a border between the groups for heuristic reasons. The expert identifies points located in the mismatch zone of the heuristic and optimization solutions and corresponding to the objects $i^{\prime}=\overline{1, I} \in \overline{1, I_{m-1, m}}$. In addition to monitoring and rating ratings about objects, an expert has additional information about them, which allows him to enter subjective ratings

$$
z_{i^{\prime}}=\left\{\begin{array}{c}
1, \text { if the } D_{i^{\prime}} \text { should be assigned to }(m-1)-\text { th group }, \\
0, \text { otherwise, } \\
i^{\prime}=\overline{1, I^{\prime}}
\end{array}\right.
$$

The coherence of the decision and assessment for objects, the expert characterizes the values of probabilities,

$$
p_{z_{i}^{\prime}}=P\left(z_{i}^{\prime} / z_{i}^{\prime}=1\right), q_{z_{i}^{\prime}}=P\left(z_{i}^{\prime} / z_{i}^{\prime}=0\right)
$$

The correction of the probability values $p_{x_{i}^{\prime}}$ according to the probability values (13) is carried out using the Bayes formula

$$
p^{k} x_{i}^{\prime}=\frac{p_{z_{i}} p_{x_{i}^{\prime}}}{p_{z_{i}^{\prime}} p_{x_{i}^{\prime}}+q_{z_{i}} q_{x_{i}^{\prime}}}, i^{\prime}=\overline{1, I^{\prime}}
$$

Then, starting from the initial values of the probabilities (14) for the variables $i^{\prime}=\overline{1, I^{\prime}}$ and the values $p^{k} x_{i}$ for the remaining variables, a randomized search and a new stage in the process of agreeing on alternative solutions for the separation of the $(m-1)$-th $m$-th groups are launched. The procedure continues until a compromise is found that suits the experts of the managing center.

\section{Optimization modeling of the scattering properties of DS formed in groups}

There is a reduction in the structure of the object in order to align the scattering properties of the DS so as to increase the level of the lower estimate $Y_{i}^{M}$. The integration mechanism is used to solve the problem: for lower-level groups, we note a certain subset of DS $D_{i}$, $(i=1, . ., \mathrm{I})$ with low integral estimates $\mathrm{Yj}(\mathrm{j}=1, . . \mathrm{J})$, they can be absorbed by the leader objects $D_{k},(k=1, . ., \mathrm{K})$ with a low RCS level $\sigma_{j}(\mathrm{j}=1, . ., \mathrm{J}), \mathrm{J}=\mathrm{K}, \sigma_{j}>>\sigma_{k}$.

The new DS, which arose as a result of the association, is characterized by an integral $\operatorname{RCS} Y_{j k}=f\left(Y_{j}, Y_{k}\right)<Y_{j},(j=\overline{1, J}, k=\overline{1, K})$. The formula for obtaining the integral RCS of a new DS, for example, can be of the form: $Y_{j k}=c_{1} Y_{j}+c_{2} Y_{k}, c_{1}+c_{2}=1, c_{1}, c_{2} \geq 0$. The problem is a discrete bicriterial optimization problem with linear constraints. When solving it, a genetic algorithm (GA) was used [9, 10].

It is necessary to ensure such an absorption by each of the objects $D_{i}$ of one of the objects $D_{k}$ in order to increase the level of the lower rating and at the same time, so that 
the total decrease in the level of the integral evaluation of the leading objects $Y_{i}^{M}$ is minimal. This setting corresponds to a bicriteria optimization model with Boolean variables:

$$
x_{i k}=\left\{\begin{array}{c}
1, \text { if } D S D_{i} \text { absorbs } D S D_{k}, \\
0, \text { otherwise }
\end{array}\right.
$$

target functions:

$$
\begin{gathered}
\sum_{i=1}^{I} \sum_{k=1}^{K}\left(Y_{i}-Y_{i k} x_{i k}\right) \rightarrow \min , \\
\min _{1 \leq i \leq I}\left(\sum_{k=1}^{K} Y_{i k} x_{i k}\right) \rightarrow \max ,
\end{gathered}
$$

and limitations:

$$
\begin{gathered}
\sum_{i=1}^{I} x_{i k}=1, k=\overline{1, K}, \\
\sum_{k=1}^{K} x_{i k}=1, i=\overline{1, I}, \\
x_{i k} \in\{0,1\}, k=\overline{1, K}, i=\overline{1, I} .
\end{gathered}
$$

Note that since estimates $Y_{i}$ in the framework of this model are constants and not variable values, the objective function (16) can be rewritten in the form:

$$
\sum_{i=1}^{I} \sum_{k=1}^{K} Y_{i k} x_{i k} \rightarrow \max .
$$

To continue the integration process within the framework of a reduced network with the number of objects $I_{1}=I-K$, a new group of leader objects $D_{i_{1}}\left(i_{1}=\overline{1, I_{1}}\right)$ is allocated and a new set of objects $O_{k_{1}}\left(k_{1}=\overline{1, K_{1}}\right), K_{1}=I_{1}$ is formed and problem (16) - (21) is solved again. With the continuation of the network reduction procedure, it is possible to absorb several objects with a low level of efficiency by the leader object.

\section{Calculation results based on proposed approaches}

We considered DS, which are square two-dimensional cylinders located at a certain distance from each other and differing in rotation angles $\alpha$ i (Fig. 1). The parameters of the problem were as follows: DS sizes $U=5 \lambda, \mathrm{L}_{1}=\ldots \mathrm{L}_{\mathrm{N}-1}=7 \lambda, \mathrm{N}=12,0^{\circ} \leq \alpha_{\mathrm{i}} \leq 20^{\circ}$, sector of observation angles $0^{\circ} \leq \varphi \leq 30^{\circ}$.. Scattered fields were calculated based on the method of integral equations. After applying the considered algorithm, we obtained three reduced groups of DS. The average RCS level in the considered sector of angles did not exceed $23 \mathrm{~dB}$. 


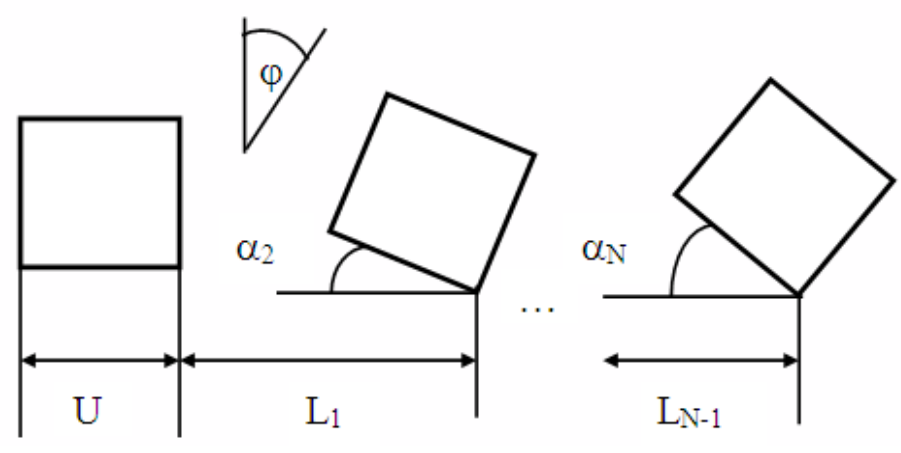

Fig. 1. The scheme of scattering of electromagnetic waves on an object consisting of many DS.

\section{Conclusion}

A combined approach is proposed on the basis of which it is possible to optimize the scattering characteristics of objects of complex shape. The results of calculations during a test numerical experiment are presented.

\section{References}

1. Ahsan Illahi, M. Bashir, Majeed A. S. Alkanhal, Sadia Khatoon, Abdul Ghaffar, Yasin Khan Electromagnetic waves scattering from a sphere of complex conjugate medium. J. Europ. Opt.1 Soc. Rap. Publ, 15(1), p.10 (2019)

2. J. Xang, S. He, Y. Zhang, G. Zhu, A spectral domain approach for thecalculation of the scattering of the stratified uniaxial electric anisotropicmedia under point source excitation with arbitrary orientation, Int. J. Appl.Electromagn. Mech. 48, pp.33-46 (2015)

3. Z. Yang, P. Gao, C. Zhang, X. Li, J. Ye, Scattering effect of the high-indexdielectric nanospheres for high performance hydrogenated amorphoussilicon thin-film solar cells, Sci. Rep., 6, p. 30503 (2016)

4. Rawaz Kurda, Jorge de Brito, Jose Dinis Silvestre, CONCRETop - A multi-criteria decision method for concrete optimization, Environmental Impact Assessment Review, 74, pp.73-85 (2019)

5. G.A. Carpenter, S. Grossberg, Adaptive Resonance Theory (Cambridge, MA: MIT Press, 2003)

6. G.A. Carpenter, S. Grossberg, D.B. Rosen, Fuzzy ART: Fast stable learning and categorization of analog patterns by an adaptive resonance system, Neural Networks, 4, pp. 759-771 (1991)

7. Igor Lvovich, Y Lvovich, Andrey Preobrazhenskiy, O. N. Choporov, Algorithmic procedures for selection control options for electric power, Systems IOP Conference Series: Materials Science and Engineering, 537, p. 062029 (2019)

8. I. Ya. Lvovich, Ya. E. Lvovich, Yu. S. Sakharov, and B. Ye. Bolshakov Solution of the multiple-choice problem based on visualization of the computational experiment and expert evaluation results, AIP Conference Proceedings, 2116, p. 200011 (2019)

9. Jr I Fister, A Iglesias, A Galvez, et al. Differential evolution for association rule mining using categorical and numerical attributes, Intell Data Eng Autom Learn, 11314, pp.79-88 (2018)

10. Ruaa Al-fallujiRuaa, Al-fallujiAdel Al-Zoghabi, Genetic Algorithms Genetic Algorithms Agenda, (2019), DOI: 10.13140/RG.2.2.24368.20489 